Alena PODHORNÁ-POLICKÁ

Université Masaryk de Brno

Anne-Caroline FIÉVET

EHESS - Paris

http://dx.doi.org/10.18778/8088-785-5.10

\title{
La circulation du néologisme SWAG : résultats d'une enquête par questionnaires auprès de jeunes de la région parisienne et de Nice
}

\section{Résumé}

L’anglicisme swag s'est propagé très rapidement ces dernières années aussi bien dans le parler des jeunes Français que dans le parler des jeunes Tchèques ou Polonais. Sa diffusion dans l'espace médiatique français et auprès de jeunes étudiants, enquêtés pour cette recherche, a pourtant ses spécificités. Elles reflètent non seulement la diversité géographique et socioculturelle de la France contemporaine mais également les résultats obtenus grâce à des méthodes appliquées à la recherche sur les néologismes (à savoir le projet européen de recherche sur les emprunts néologiques EmpNeo vs un projet tchéco-français de recherche sur les néologismes identitaires pour les jeunes - Sociolex). Le présent article cible la polysémie dans la langue de départ et dans la langue cible de l'emprunt (anglais / français), ainsi que l'histoire récente de la dictionnairisation du néologisme. Il présente également quelques résultats d'une méthode longitudinale, Sociolex, effectuée entre 2013 et 2015 en région parisienne et à Nice.

Mots-clés : néologie, français, anglicisme, diffusion, lexicographie, méthodologie 


\section{Summary}

The English polysemic loanword swag is nowadays spreading to young French people's language, as well as to other European languages studied in the project EmpNeo (neologic loanwords) - mainly for Polish and Czech. The French diffusion has its own specificities in media as well as in the communication between peers. The latter reflects geographical and sociocultural diversity of contemporary France. But the results also reflect the features of applied methodology for research on neology (EmpNeo and Sociolex, a French-Czech research project on identitary words of youth). The paper focuses on the polysemy of swag in English and in French, the recent history of its lexicographic occurrences and presents some results of the longitudinal methods of Sociolex between 20132015 in the area of Paris and in Nice (South of France).

Keywords: neology, French, anglicism, diffusion, lexicography, methodology

\section{Introduction}

Menant des recherches depuis plus de dix ans sur la circulation du lexème bo$\operatorname{los}(s / \mathrm{se})$, nous avons rencontré plusieurs fois le lexème swag comme étant son opposé dans la hiérarchie des bandes de jeunes qui se classifient en trois catégories : populaire, bolos(s/se) et swag. Le néologisme swag (tout du moins par rapport à l'ancienneté de bolos(s/se) dans le parler des jeunes en France) nous intéresse également car il a fait récemment la même irruption dans le vocabulaire des jeunes Tchèques. Il devient ainsi un « mot identitaire (Podhorná-Polická 2010) prototypique à la fois en français et dans les autres langues étudiées dans le cadre du projet EmpNeo (à savoir le français, le polonais et le tchèque), ce dont témoignent des fiches descriptives effectuées pour le lexème swag dans les trois langues du projet (pour la méthodologie détaillée de ce projet, voire Hildenbrand, Kacprzak et Sablayrolles (éds.) 2015 ; ou encore Podhorná-Polická et Viaux, à paraître). 


\section{Emprunt néologique à l'anglais : généralités et sémantisme d'un mot polysémique}

Déjà en anglais, swag est un mot polysémique et substandard : le dictionnaire anglais-tchèque del'anglais substandard Wazzap? (2008) en donne la définition suivante « 1 . butin (des voleurs, etc.), 2. sacoche (porté par les vagabonds) (en anglais de l'Australie) ».

L'anglicisme swag s'est répandu très rapidement depuis le début des années 2010 via les médias et la publicité et nous avons souhaité étudier de plus près cette fulgurante ascension. Contrairement aux néologismes décrivant une réalité toute nouvelle, l'émergence lexicale d'un lexème tel que swag va de pair avec la concurrence que le nouveau mot a avec ses équivalents sémantiques (ici : «stylé», «qui a du style», «à la mode»).

Avec la propagation de la nouveauté, le système bouge tant du point de vue onomasiologique que sémasiologique (centre-périphérie en fonction de la fréquence, spécification-élargissement sémantique), phénomène perceptible de façon générale pour tous les néologismes identitaires où swag ne représente pas une exception à la règle.

Une autre généralité concernant les emprunts néologiques est que ces derniers sont souvent « traduits » en co-texte par reformulation, faisant ainsi apparaître leur(s) équivalent(s). Ceci s'opère en reprise soit cataphorique soit anaphorique du mot supposé ne pas faire partie du lexique du destinataire envisagé de l'énoncé. Pour swag par exemple, le film grand public Les Profs (2013, de Pierre-François Martin-Laval) montre bien le souci des réalisateurs d'expliquer le néologisme identitaire pour les jeunes aux spectateurs d'autres générations. En effet, on peut tout d'abord relever l'expression synonymique « avoir trop la classe » ( Ils ont trop la classe » - un élève à un autre élève en parlant de leurs professeurs - 1 heure 15 minutes 17 secondes) puis, quelques minutes plus tard, sur le même modèle, «avoir trop l'swag " («Franchement, les profs, ils ont trop l'swag » - un élève à un journaliste - 1 heure 18 minutes 45 secondes).

Une troisième généralité concernant les emprunts néologiques à caractère identitaire pour les jeunes consiste dans le fait qu'il est difficile de repérer la période de son adoption par la langue cible étant donné que cette adoption s'opère différemment d'un lieu / réseau communicatif à l'autre. Ce repérage est d'autant plus difficile si le mot est polysémique, la diffusion d'une acception peut être plus précoce, massive qu'une autre (cf. Pergnier, 1989 : 121-156). 


\section{L'apparition de swag dans les dictionnaires}

À partir de 2012, swag apparaitt progressivement dans les dictionnaires de français spécialisés : il est présent dans le dictionnaire officiel du Parlement européen, Le dictionnaire multilingue des mots nouveaux (2013: 176), avec la définition suivante : « (anglicisme) qui a du style, qui est charismatique. » Il apparaît également dans les dictionnaires popularisant la néologie comme 365 mots nouveaux expliqués (2013:212), où il est mentionné que « le swag, c'est ce petit style naturel qui fait la différence $»$.

Il fait également son apparition dans les dictionnaires d'argot commun des jeunes comme le Dictionnaire ados français (2014: 319: avoir le swag, être swag, être trop swag) : « expression anglo-saxonne pouvant s'utiliser à la fois en tant que nom commun ou qu'adjectif qualificatif, et désignant une allure générale plutôt cool, stylée, limite voyou mais classe en toutes circonstances ». Enfin, il est également intégré à des dictionnaires d'argot commun des jeunes des cités comme Tout l'argot des banlieues (2013: 518, swag $(g)$, swagué - présent dans la version en ligne depuis 2012 selon son administrateur Cobra le cynique avec la définition suivante : «style vestimentaire, façon d'être »).

Néologisme bien documenté dans les dictionnaires, swag est désormais candidat à un passage dans les dictionnaires usuels : il apparaît dans la section «Mots nouveaux» du Dictionnaire Hachette à partir de la version de 2014 (parue en juin 2013) et y est toujours présent dans la version de 2017 (il n’a pas intégré le corps du dictionnaire) avec la définition suivante : « comportement, façon d'être dénotant une certaine autorité naturelle ».

\section{Deux méthodologies à comparer pour étudier la diffusion du lexème swag}

Coordonné par Jean-François Sablayrolles, le projet EmpNeo est un projet franco-polono-tchèque, qui a pour but d'observer l'insertion des emprunts néologiques (quasi exclusivement des anglicismes) dans la société de plusieurs pays européens pendant une période donnée via des occurrences dans les archives de presse (corpus journalistique donc écrit), dans des corpus représentatifs des langues étudiées et dans des dictionnaires traditionnels et en ligne. Pour swag, qui fait partie des emprunts néologiques choisis par l'équipe EmpNeo pour un traitement sous forme de fiches en 2016 , on a pu constater une fulgurante dif- 
fusion dans l'espace médiatique ainsi que de riches familles dérivationnelles en français et en tchèque. Pour des raisons de taille, nous reprenons seulement des extraits de la fiche française à la fin de cet article avec l'aimable autorisation de son auteure, Inès Djazi.

La méthodologie Sociolex est une méthode longitudinale par questionnaires distribués tous les ans auprès des étudiants de licence de plusieurs universités. Elle vise les déclarations d'usage et de circulation, les avis sur le sentiment néologique et sur l'entrée potentielle dans les dictionnaires usuels. Nous avons adopté cette méthodologie depuis une dizaine d'années (recherches sur la circulation du lexème bolos $(\mathrm{s} / \mathrm{se}$ ) depuis 2008, cf. Fiévet-Podhorná et Polická 2009, etc.). L'âge est une constante (premières années d'université), ceci étant notamment dû à un manque de moyens pour effectuer des enquêtes englobant toutes les tranches d'âge de la société. La méthodologie Sociolex vise l'oral et la néologie identitaire (en quelque sorte la partie immergée de l'iceberg de la diffusion par les réseaux (sociaux) de pairs), ce qui est moins visible avec la méthodologie par fiches du groupe EmpNeo, dont ce n'est pas l'objectif : les deux méthodes s'avèrent complémentaires pour le français (les deux projets étant très différents).

\section{Enquête : déroulement et quelques résultats}

La méthodologie Sociolex a été appliquée au lexème swag dès 2013. Elle a consisté en deux étapes à un intervalle de presque deux ans (entre décembre 2013 et février 2014 pour la première étape et décembre 2015 pour la deuxième étape) pour un total de 274 questionnaires recueillis, dont 203 de la région parisienne. En effet, afin d'observer une éventuelle variation diatopique, le questionnaire a également été distribué à 71 étudiants de l'Université Nice Sophia Antipolis.

Le tableau 1 montre les résultats globaux des déclarations des jeunes concernant leur connaissance et/ou utilisation du lexème swag en fonction des lieux et des périodes d'enquête. Si l'on considère la première période d'enquête (décembre 2013-février 2014) qui a pu être réalisée dans trois lieux (Paris Descartes, Créteil et Nice), on ne relève pas de différence sensible entre les déclarations des jeunes étudiants de Paris Descartes et de Nice puisqu'environ un tiers des jeunes déclarent l'utiliser, deux tiers déclarent le connaître sans l'utiliser et un seul étudiant déclare ne pas le connaître (un étudiant de 23 ans à Paris Descartes). Ce résultat, qui devra être consolidé avec des enquêtes ultérieures, permet d'avancer l'hypothèse qu'il n'y a pas de variation diatopique pour swag 
entre la région parisienne et le sud de la France (comme c'est par exemple le cas de bolos(s/se) qui est un lexème issu de l'Île-de-France et qui s'est propagé ensuite dans les autres régions). Bien que le nombre de questionnaires recueillis pour l'Université Paris-Est Créteil soit seulement de 25, les résultats montrent une tendance intéressante, à savoir que les jeunes ont massivement déclaré le connaître ( $88 \%$; seul un étudiant de 36 ans a déclaré ne pas le connaître) mais ils sont peu nombreux à avoir déclaré l'utiliser (8\%), comme s'il ne faisait pas partie de leur argot ou/et qu'il était déjà passé de mode pour eux. L'Université Paris-Est Créteil se trouvant en banlieue parisienne, les étudiants issus des quartiers d'habitat social y sont nombreux. Ainsi, on peut postuler, en comparant les résultats obtenus d'un côté à Paris Descartes / Nice et de l'autre à Créteil, que swag est plutôt un lexème de l'argot commun des jeunes (argot de type générationnel) mais n'est pas ou n'est plus un lexème de l'argot commun des jeunes des cités (argot de type sociologique).

Tableau 1. Déclarations sur l'usage ou la connaissance du lexème swag en fonction du site et de la période d'enquête

\begin{tabular}{|c|c|c|c|c|c|}
\hline & $\begin{array}{c}\text { Paris Descartes } \\
\text { déc. 2013 } \\
\text { et fév. 2014 }\end{array}$ & $\begin{array}{c}\text { Créteil } \\
\text { janv. 2014 }\end{array}$ & $\begin{array}{c}\text { Nice } \\
\text { fév. 2014 }\end{array}$ & $\begin{array}{c}\text { Paris } \\
\text { Descartes } \\
\text { déc. 2015 }\end{array}$ & Total \\
\hline oui A & $31 \%(26)$ & $8 \%(2)$ & $37 \%(26)$ & $26 \%(24)$ & $\mathbf{2 8 \% ( 7 8 )}$ \\
\hline oui P & $67 \%(57)$ & $88 \%(22)$ & $62 \%(44)$ & $62 \%(58)$ & $\mathbf{6 6 \% ( 1 8 1 )}$ \\
\hline non & $1 \%(1)$ & $4 \%(1)$ & $0 \%(0)$ & $12 \%(11)$ & $\mathbf{5 \% ( 1 3 )}$ \\
\hline 0 & $1 \%(1)$ & $0 \%(0)$ & $1 \%(1)$ & $0 \%(0)$ & $\mathbf{1 \% ( 2 )}$ \\
\hline Total & $\mathbf{1 0 0 \% ( 8 5 )}$ & $\mathbf{1 0 0 \% ( 2 5 )}$ & $\mathbf{1 0 0 \% ( 7 1 )}$ & $\mathbf{1 0 0 \% ( 9 3 )}$ & $\mathbf{1 0 0 \% ( 2 7 4 )}$ \\
\hline
\end{tabular}

Légende pour les tableaux :

P5 : Université Paris Descartes (anciennement Paris 5 - René Descartes)

Créteil : Université Paris Est - Créteil

Nice : Université Nice Sophia Antipolis

oui $\mathrm{A}$ : déclaration d'usage active du lexème

oui $\mathrm{P}$ : déclaration de connaissance passive du lexème

non : déclaration de non-connaissance du lexème

0 : aucune déclaration ou déclaration ininterprétable

Si l'on compare ces résultats à ceux de la deuxième période (décembre 2015), donc aux résultats des questionnaires distribués deux ans plus tard à l'Université Paris Descartes, on constate une baisse de l'utilisation (passant de $31 \%$ pour 
Paris Descartes fin 2013-début 2014 à $26 \%$ dans la même université fin 2015). Le nombre d'étudiants qui déclarent le connaître mais ne pas l'utiliser baisse également (de $67 \%$ à $62 \%$ ). De plus, le nombre d'étudiants déclarant ne pas le connaître augmente sensiblement (de $1 \%$ à $12 \%$ ). Parmi les 11 étudiants qui ont déclaré ne pas le connaître, on trouve un étudiant de 25 ans, six étudiants étrangers mais également quatre étudiantes ayant un profil « classique» (entre 18 et 23 ans, ayant habité en France toute leur vie), ce qui laisse penser que le lexème a, entre fin 2013 et fin 2015, déjà connu un certain recul, tout au moins dans le lexique identitaire des jeunes.

Puisque nous avons postulé que swag était un lexème a priori générationnel (avec une connotation "branché » pour son origine anglo-saxonne dans certains milieux adultes), observons maintenant les résultats des déclarations sur sa connaissance ou son utilisation en fonction de l'âge des enquêtés.

Tableau 2. Déclarations sur l'usage ou la connaissance du lexème swag en fonction de l'âge des enquêtés (pour les quatre enquêtes, entre décembre 2013 et décembre 2015)

\begin{tabular}{|c|c|c|c|c|c|c|c|c|c|c|c|}
\hline & 17 & 18 & 19 & 20 & 21 & 22 & 23 & 24 & $\begin{array}{l}25 \\
\text { et }+\end{array}$ & $\begin{array}{l}\text { Sans } \\
\text { indication } \\
\text { d'âge }\end{array}$ & Total \\
\hline oui $A$ & $\begin{array}{c}33 \% \\
\text { (1) }\end{array}$ & $\begin{array}{c}34 \% \\
(20)\end{array}$ & $\begin{array}{c}35 \% \\
(18)\end{array}$ & $\begin{array}{c}24 \% \\
(15)\end{array}$ & $\begin{array}{c}33 \% \\
(14)\end{array}$ & $\begin{array}{c}21 \% \\
(4)\end{array}$ & $\begin{array}{c}18 \% \\
(3)\end{array}$ & $\begin{array}{c}20 \% \\
\text { (1) }\end{array}$ & $\begin{array}{c}0 \% \\
(0)\end{array}$ & (2) & (78) \\
\hline oui $P$ & $\begin{array}{c}66 \% \\
\text { (2) }\end{array}$ & $\begin{array}{c}64 \% \\
(38)\end{array}$ & $\begin{array}{c}63 \% \\
(33)\end{array}$ & $\begin{array}{c}71 \% \\
(44)\end{array}$ & $\begin{array}{c}64 \% \\
(27)\end{array}$ & $\begin{array}{c}68 \% \\
(13)\end{array}$ & $\begin{array}{c}71 \% \\
(12)\end{array}$ & $\begin{array}{c}80 \% \\
\text { (4) }\end{array}$ & $\begin{array}{c}64 \% \\
(7)\end{array}$ & $\begin{array}{c}25 \% \\
\text { (1) }\end{array}$ & $\begin{array}{l}66 \% \\
\text { (181) }\end{array}$ \\
\hline non & $\begin{array}{c}0 \% \\
(0)\end{array}$ & $\begin{array}{l}0 \% \\
(0)\end{array}$ & $\begin{array}{c}2 \% \\
\text { (1) }\end{array}$ & $\begin{array}{c}5 \% \\
(3)\end{array}$ & $\begin{array}{r}3 \% \\
\text { (1) }\end{array}$ & $\begin{array}{c}11 \% \\
(2)\end{array}$ & $\begin{array}{c}11 \% \\
\text { (2) }\end{array}$ & $\begin{array}{c}0 \% \\
(0)\end{array}$ & $\begin{array}{c}36 \% \\
(4)\end{array}$ & $\begin{array}{c}0 \% \\
\text { (0) }\end{array}$ & $\begin{array}{l}5 \% \\
(13)\end{array}$ \\
\hline 0 & $\begin{array}{l}0 \% \\
(0)\end{array}$ & $\begin{array}{l}2 \% \\
\text { (1) }\end{array}$ & $\begin{array}{l}0 \% \\
(0)\end{array}$ & $\begin{array}{l}0 \% \\
(0)\end{array}$ & $\begin{array}{l}0 \% \\
(0)\end{array}$ & $\begin{array}{l}0 \% \\
(0)\end{array}$ & $\begin{array}{l}0 \% \\
(0)\end{array}$ & $\begin{array}{l}0 \% \\
(0)\end{array}$ & $\begin{array}{l}0 \% \\
\text { (0) }\end{array}$ & $\begin{array}{c}25 \% \\
\text { (1) }\end{array}$ & $\begin{array}{l}1 \% \\
\text { (2) }\end{array}$ \\
\hline Tc & $\begin{array}{l}100 \% \\
\text { (3) }\end{array}$ & $\begin{array}{c}100 \% \\
(59)\end{array}$ & $\begin{array}{c}100 \% \\
(52)\end{array}$ & $\begin{array}{c}100 \% \\
(62)\end{array}$ & $\begin{array}{c}100 \% \\
(42)\end{array}$ & $\begin{array}{c}100 \% \\
(19)\end{array}$ & $\begin{array}{c}100 \% \\
(17)\end{array}$ & $\begin{array}{c}100 \% \\
\text { (5) }\end{array}$ & $\begin{array}{c}100 \% \\
\text { (11) }\end{array}$ & $\begin{array}{c}100 \% \\
\text { (4) }\end{array}$ & $\begin{array}{c}100 \% \\
(274)\end{array}$ \\
\hline
\end{tabular}

Ce tableau prend en compte la totalité des questionnaires, sans distinction des périodes et des lieux. Pour 17 ans et pour 24-36 ans, le nombre de questionnaires n'est pas assez important pour pouvoir établir des pourcentages (qui ne sont donc ici qu'indicatifs). La moyenne d'âge totale est de 20,19 ans. Les résultats vont dans le sens d'un lexème générationnel puisqu'on peut observer que swag est connu voire utilisé par la majorité des jeunes entre 17 et 
24 ans. En revanche, aucun des plus de 25 ans ne déclare l'utiliser activement.

Une autre hypothèse évoquée supra est que le lexème swag serait progressivement en train de passer de mode. Dans le questionnaire, nous demandons aux étudiants (à partir du moment où ils ont déclaré qu'ils connaissent le lexème) s'ils le considèrent plutôt vieilli, stable, assez moderne ou moderne.

Tableau 3. Déclarations sur la modernité du lexème swag en fonction du site et de la période d'enquête (pour les enquêtés ayant déclaré l'utiliser activement ou le connaître passivement)

\begin{tabular}{|l|c|c|c|c|c|} 
& $\begin{array}{c}\text { P5 } \\
\text { 2013-2014 }\end{array}$ & $\begin{array}{c}\text { Créteil } \\
\mathbf{2 0 1 4}\end{array}$ & $\begin{array}{c}\text { Nice } \\
\mathbf{2 0 1 4}\end{array}$ & $\begin{array}{c}\text { P5 } \\
\mathbf{2 0 1 5}\end{array}$ & Total \\
\hline vieilli & $3 \%(2)$ & $8 \%(2)$ & $7 \%(5)$ & $11 \%(9)$ & $\mathbf{1 8}$ \\
\hline stable & $11 \%(9)$ & $11 \%(2,5)$ & $8 \%(5,5)$ & $26 \%(21)$ & $\mathbf{3 8}$ \\
\hline assez moderne & $37 \%(31)$ & $\mathbf{5 6 \% ( 1 3 , 5 )}$ & $37 \%(26)$ & $\mathbf{4 6 \% ( 3 8 )}$ & $\mathbf{1 0 8 , 5}$ \\
\hline moderne & $\mathbf{4 8 \% ( 4 0 )}$ & $17 \%(4)$ & $\mathbf{4 6 \% ( 3 2 , 5 )}$ & $15 \%(12)$ & $\mathbf{8 8 , 5}$ \\
\hline 0 & $1 \%(1)$ & $8 \%(2)$ & $2 \%(1)$ & $2 \%(2)$ & $\mathbf{6}$ \\
\hline Total & $\mathbf{1 0 0 \% ( 8 3 )}$ & $\mathbf{1 0 0 \% ( 2 4 )}$ & $\mathbf{1 0 0 \% ( 7 0 )}$ & $\mathbf{1 0 0 \% ( 8 2 )}$ & $\mathbf{2 5 9}$ \\
\hline
\end{tabular}

Le tableau 3 montre les résultats à cette question en fonction des sites et des périodes d'enquête. Il arrive que des enquêtés aient coché deux réponses, auquel cas il a été décidé de compter 0,5 pour chacune des réponses (ce qui explique la présence de certains chiffres (avec des décimales). Les résultats permettent d'établir un lien entre modernité déclarée et utilisation active. D’un côté, les étudiants de Paris Descartes 2013-2014 et de Nice 2014, qui sont les plus nombreux à avoir déclaré utiliser le lexème, sont nombreux à déclarer qu'il est plutôt moderne ( $48 \%$ pour Paris Descartes et $46 \%$ pour Nice). De l'autre côté, les étudiants de Créteil 2014 et de Paris Descartes 2015, pour lesquels l'utilisation active est moins importante, déclarent dans une forte proportion que le lexème est plutôt assez moderne (56 \% pour Créteil 2014 et $46 \%$ pour Paris Descartes 2015). Ainsi, on peut postuler que les étudiants de Créteil avaient déjà constaté début 2014 que le lexème était en train de passer de mode, ce qui est visible seulement deux ans plus tard chez les étudiants de Paris Descartes (fin 2015). Une hypothèse peut être établie à ce propos, à savoir que le lexème swag a été un mot identitaire pour les jeunes des cités (véhiculé via le rap, notamment grâce au rappeur La Fouine qui, en 2009, a lancé une marque vestimentaire du nom de "Swagg ") mais que, tout comme pour bo$\operatorname{los}(s / s e)$, sa propagation massive vers d'autres milieux socioéconomiques et 
dans l'espace médiatique en général a eu pour conséquence le fait que, pour ces jeunes, sa modernité / son expressivité se sont estompées au profit d'autres mots identitaires.

\section{En guise de conclusion}

La corrélation entre les différentes questions posées dans les questionnaires de Sociolex montre bon nombre d'autres résultats intéressants permettant d'établir des hypothèses sur les vecteurs de diffusion qui s'avèrent être nombreux et variés (communications entre pairs ou entre camarades de classe, écoute des chansons des interprètes préférés ou des tubes que les radios émettent en boucle). Comme swag est un anglicisme identitaire, les étudiants questionnés ont donné des pistes tous azimuts mélangeant le monde anglophone (p. ex. le chanteur pop Justin Bieber) et le monde francophone (p. ex. le rappeur La Fouine), ce qui nous permet de nous interroger sur les frontières entre le global et le local en matière d'emprunts à l'anglais, qu'il s'agisse du sémantisme du néologisme ou des activités épilinguistiques qui influencent les enquêtés mais aussi les chercheurs des deux projets exposés.

\section{Références bibliographiques}

Calvet Louis-Jean, 2013, "Le bolosse n’a pas le swag », Le français dans le monde, en ligne, disponible sur, http://www.fdlm.org/blog/2013/05/08/lebolosse-na-pas-le-swag/, (consulté le 1/10/2016).

Cheminée Pascale \& Weber Romain, 2013, Le grand quiz de la langue française, Paris, Le Monde.

Desalmand Paul \& Stalloni Yves, 2013, 365 mots nouveaux expliqués, Paris, Éditions du Chêne.

Dictionnaire Hachette, 2012-2017, Vanves, Hachette livre.

Hildenbrand Zuzana, Kacprzak Alicja, Sablayrolles Jean-François (éds.), 2015, Emprunts néologiques et équivalents autochtones en français, en polonais et en tchèque, Limoges, Lambert Lucas, coll. «La Lexicothèque ».

Parlement européen, Direction générale de la traduction, 2013, Dictionnaire multilingue des mots nouveaux, $4^{\mathrm{e}}$ édition, Luxembourg, Union Européenne.

Pergnier Maurice, 1989, Les anglicismes, Paris, PUF. 
Podhorná-Polická Alena, Fiévet Anne-Caroline, 2009, «À la recherche de la circulation d'un néologisme identitaire : le cas de bolos », in Standard et périphéries de la langue, Kacprzak Alicja, Goudaillier Jean-Pierre, Łódź -Łask, Oficyna Wydawnicza Leksem, p. 207-223.

Podhorná-Polická Alena, 2010, «Expressivité des «mots identitaires〉 dans l'argot des jeunes », in Actes du XXVe Congrès International de Linguistique et de Philologie Romanes. Innsbruck 2007 [3-8 septembre 2007, Innsbruck], Iliescu Maria, Siller-Runggaldier Heidi, Danler Paul (éds.), Tome IV, Berlin-New York, Mouton de Gruyter, p. 213-222.

Podhorná-Polická Alena, Viaux Julie, à paraître, « lexique des réseaux sociaux : étude franco-tchèque des emprunts à l'anglais et de leurs équivalents autochtones ", in Lexique : néologie, emprunt, sémantique, Ieda Maria Alves, Salah Mejri et Jean-François Sablayrolles (éds.), São Paulo, Humanitas.

Ribeiro Stéphane, 2014, Dictionnaire ados français, Paris, éditions First.

Tengour Abdelkarim, 2013, Tout l'argot des banlieues, Paris, Les éditions de l'Opportun.

Wazzap ? slovník slangu a hovorové angličtiny, 2008, Brno, Lingea. 


\section{Annexe : Extraits de la fiche fr_swag réalisée par Inès Djazi, projet EmpNeo}

\section{Swag ID 10/01/2016}

2F. Définition

- Style vestimentaire, branché, associé à une attitude « cool » $(\mathrm{N})$

- Ayant un style décontracté, cool (Adj)

\section{AF. EMPRUNT}

\begin{tabular}{|c|c|}
\hline $\begin{array}{l}\text { AF4. Dictionnaires } \\
\text { sur la toile }\end{array}$ & $\begin{array}{l}\text { Wiktionnaire : De l'anglais « swag ", diminutif de "swagger » } \\
\text { Étymologie : langage populaire indiquant que swag serait un } \\
\text { acronyme signifiant Secretly We Are Gay « secrètement nous } \\
\text { sommes gay " }\end{array}$ \\
\hline $\begin{array}{l}\text { AF5. Contextes } \\
\text { avec références }\end{array}$ & $\begin{array}{l}\text {-Télé-Loisirs.fr : Employé constamment par Nabilla dans Les } \\
\text { Anges } 5 \text { (NRJ12), le terme "swag" est décliné à toutes les sauces. } \\
\text { Utilisé comme une expression, un adjectif et même un verbe. http:// } \\
\text { www.programme-tv.net/news/buzz/38741-que-veut-dire-swag/ } \\
\text { - Site web dédié aux achats de vêtements : http://realnswag. } \\
\text { fr/shop/fr/ }\end{array}$ \\
\hline \multicolumn{2}{|r|}{ AF6. Mesures de diffusion et acclimatation } \\
\hline $\begin{array}{l}\text { AF6.1. Nombre de } \\
\text { pages sur la toile }\end{array}$ & 439000 résultats de français de France. \\
\hline $\begin{array}{l}\text { AF6.2. Archives de } \\
\text { presse }\end{array}$ & $\begin{array}{l}\text { Libération : } 21 \text { résultats. Première occurrence le 30/07/2011 et } \\
\text { la plus récente remonte au } 31 / 10 / 2015 \text {. Deux occurrences avec } \\
\text { l'auxiliaire « avoir » et une seul avec l'auxiliaire « être » (être swag). } \\
\text { - Le 30/07/2011 } \\
\text {...née pour désigner de ravissantes idiotes richissimes, cette } \\
\text { façon de voir des « it » partout a pris des allures d'avalanche. } \\
\text { Tout est « it ». Et pas seulement le blog de Paris Hilton. It-bag, } \\
\text { it-blush, it-tongs, it-music, it-taboulé, it-it (à ne pas confondre, } \\
\text { bien que it ethnique, avec hittite). Il y a peu, on disait « must ». } \\
\text { Mais depuis qu'on le dit aussi dans les émissions de télé-achat, } \\
\text { il fallait trouver autre chose. L'été « moderne » sera it ou ne sera } \\
\text { pas. Et Rimbaud, it-poète notoire, pourrait écrire : « II faut être } \\
\text { résolument it. » En français, puisque le « it », c'est « ça », on } \\
\text { peut parler de ça-fille ou de ça-sac. } \\
\text { It et tragédie : aux dernières nouvelles des cervelles de per- } \\
\text { ruches, le « swag », qui en anglais désigne l'allure, serait en } \\
\text { passe de détrôner le it. Total : le swag est it. }\end{array}$ \\
\hline
\end{tabular}




\begin{tabular}{|c|c|}
\hline & $\begin{array}{l}\text { - le 31/07/2015- Wallerand de Saint-Just, vieil ours brun } \\
\text { Domaine de la politique en essayant de décrire un représentant } \\
\text { du FN, et secrétaire privé de Jean-Marie Le Pen. Décrivant son } \\
\text { look comme swag : (Au jeu de la phrase de campagne la plus } \\
\text { insolite, Wallerand de Saint-Just a pris une longueur d'avance. } \\
\text { Le } 12 \text { septembre, sur France } 3 \text {, la tête de liste du FN en lle- } \\
\text { de-France commente l'un de ses gadgets militants : un tee- } \\
\text { shirt affichant, sur fond bleu, sa barbe et ses lunettes à grosse } \\
\text { monture, soit deux items en vogue chez les jeunes urbains } \\
\text { branchés. "Est-ce l'électorat hipster que vous ciblez en lle- } \\
\text { de-France ? " questionne le journaliste. " Non, c'est l'électorat } \\
\text { swag que je vise, répond son invité, pince-sans-rire et cheval- } \\
\text { ière au doigt. Celui qui est détendu et un peu décontracté. ") } \\
\text { Le monde (11 résultats) du 1/06/2012 au 29/01/2016 } \\
\text { LE MONDE | } 1 \text { juin } 2012 \\
\text { Vont-ils me répartir qu'avoir le SWAG, c'est "avoir du style" et } \\
\text { me renvoyer dans les cordes avec mon Songe éculé ? } \\
\text { - Dernière occurrence 29/01/2016: Les épisodes " Jay Z vs } \\
\text { Kanye West ", "Maintenant le mariage, c'est swag ", } \\
14 / 02 / 2015 \text { dans culture et idées dont le titre est Swagg Man, } \\
\text { le corps comme capital }\end{array}$ \\
\hline & $\begin{array}{l}\text {... Dès ses débuts, il exhibe son swag, un terme alors remis au } \\
\text { goût du jour aux Etats-Unis désignant le « style ", le cool. Son } \\
\text { sourire de gosse, son ingénuité primaire, ses poses sans déco- } \\
\text { rum au milieu des grosses coupures et des bijoux en or attisent } \\
\text { la curiosité. "Le lendemain, j'ai appuyé sur le bouton "moné- } \\
\text { tiser" de YouTube. " Cinq ans après, il a } 50 \text { millions de vues } \\
\text { sur sa page officielle (certifiée) et des publicités accompagnent } \\
\text { ses vidéo - de la litière pour chat aux sites de rencontres. Il est } \\
\text { devenu l'icône immature d'une société angoissée dont l'argent } \\
\text { est la valeur suprême. }\end{array}$ \\
\hline $\begin{array}{l}\text { AF6.3. Types de } \\
\text { documents }\end{array}$ & $\begin{array}{l}\text { - Pages qui renvoient à l'émission de télé-réalité de Nabila. } \\
\text { - Sites de ventes de vêtements de tendances « Real N Swag " } \\
\text { http://realnswag.fr/shop/fr/ } \\
\text { - sites qui vendent le swag bag australien (sac de couchage) } \\
\text { - Blogs de modes et des magazines tendances mode. } \\
\text { - Presse féminine. }\end{array}$ \\
\hline
\end{tabular}

Remarques / commentaires

- swag peut-être tantôt substantif « t’as du swag », tantôt adjectif « il est swag ».

- Ce terme s'emploie souvent avec l'auxiliaire « avoir », qui veut dire « avoir le swag » son origine dériverait de l'anglais « to swagger ». 